\title{
Motion in depth: Adequate and inadequate simulation
}

\author{
R. GRAY and D. REGAN \\ York University, North York, Ontario, Canada
}

\begin{abstract}
We measured errors in estimating the absolute time to collision with a simulated approaching textured object. The texture elements were circular bright dots. When we matched the rate of angular expansion of the simulated object, the rate of expansion of the texture dots, and the rate of increase of dot separation, so as to accurately simulate an approaching object, errors were small underestimations that were independent of dot size (mean of 3.2\%). When dot angular size was held constant during the simulated approach, errors were the same as when the simulation was accurate, provided that dot size was less than 2.2-4.4 min of arc. As dot size was progressively increased, errors changed to overestimations. For the largest dot size used ( $10.5 \mathrm{~min}$ of arc at time $t=0)$, time to collision was overestimated by up to $21 \%$. A sufficiently large overestimation would mean that measures taken to avoid collision would be too late. We suggest that the relevance to everyday life of data on the perception of motion in depth and self-motion collected using constant-sized dot displays might be questionable if dot size exceeds $2.2-4.4$ min of arc.
\end{abstract}

In many investigations of the perception of an object's motion in depth and also in many investigations of heading judgment, the visual environment has been simulated by a pattern of dots whose angular size remained constant. In the everyday world of textured objects, however, both the angular size of an object and the angular size of each of the texture elements that cover the object's surface increase as the observing eye moves closer to the object. This paper discusses whether this reality limits the relevance to everyday life of the psychophysical data on motion in depth and heading that has been collected using dots of constant size.

In previous studies, we used the stimulus illustrated in Figure 1 to investigate the perception of motion in depth produced by isotropic expansion of an object's retinal image (Beverley \& Regan, 1983; Vincent \& Regan, 1997). Figures $1 \mathrm{~A}-1 \mathrm{C}$ show how the size of the simulated square object expanded while the size of the texture elements expanded in the locked mode characteristic of the everyday environment (Figure 1B) expanded more quickly than the size of the square (Figure $1 \mathrm{~A}$ ), or remained constant (Figure 1C). We found that, when texture element size re-

This research effort was sponsored by the Air Force Office of Scientific Research, Air Force Materiel Command, U.S.A.F. under Grant F49620-97-1-0051 and was supported by the National Sciences and Engineering Research Council of Canada (NSERC). D.R. holds the NSERC/CAE Industrial Research Chair of Vision and Aviation. We thank Hua Hong for writing the display software used in this study. Dr. Hong is supported by CRESTech, York University. We thank Stephanie Hajdur and Kitty Shapiro for acting as observers and Derek Harnanansingh for assistance in preparing this manuscript. Correspondence concerning this article should be addressed to D. Regan, Department of Psychology, York University, 4700 Keele St., North York, ON M3J 1P3, Canada (e-mail: dregan@yorku.ca) mained constant, the motion-in-depth mechanism was only weakly excited (Beverley \& Regan, 1983) and the absolute time to collision with the simulated approaching object was overestimated (Vincent \& Regan, 1997). Braunstein (personal communication, 1996) pointed out that these findings are not necessarily relevant to conclusions based on studies in which constant-sized dot displays were used, because both the texture element size and the separation between the centers of the texture elements remained constant in Figure 1C, whereas, in most studies using constant-sized dots, the separation between the dots expanded appropriately. In the present study, we address Braunstein's comment by using a textured target for which the size of the target, the size of the texture elements, and the separation of the texture elements could be varied independently.

\section{GENERAL METHOD}

\section{Apparatus}

A square array of bright circular dots was generated by a PC (Dell Dimension XPS, Pentium $100 \mathrm{MHz}$ with $30 \mathrm{MB}$ RAM) and displayed on a computer monitor running at 30 frames/sec with a resolution of 600 (horizontal) $\times 480$ pixels. The display (Dell Ultrascan ISTE monitor) was viewed binocularly from $580 \mathrm{~cm}$, at which distance it subtended $2.7^{\circ} \times 2.1^{\circ}$. The luminance profile along any diameter of any given dot was a Gaussian wave form. The following three variables could be manipulated independently: the side length $\left(\theta^{\circ}\right)$ of the square array of dots, dot diameter $(s)$, and dot separation $(x)$. The total number of dots in the array depended on dot size, dot separation, and the mode of expansion, as is described below.

For a rigid nonrotating object that is moving directly toward the eye at constant speed, it has been shown that

$$
T \approx \theta /(\mathrm{d} \theta / \mathrm{d} t)
$$

(Hoyle, 1957; Lee 1976), where $T$ is the time to collision with the object and $\theta$ is the object's instantaneous angular subtense. 
A

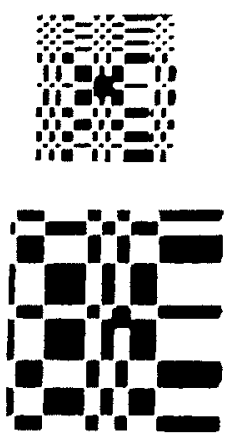

\section{B}
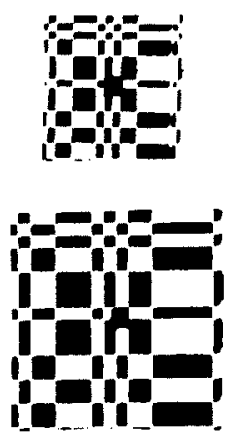
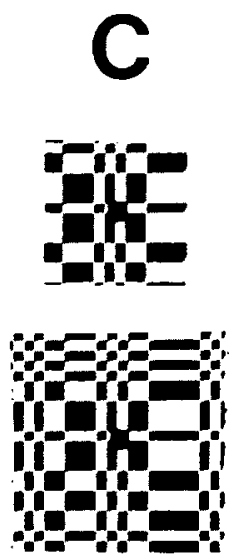

Figure 1. Textured stimuli used by Beverley and Regan (1983) and by Vincent and Regan (1997). Top row: start of presentation ramp. Bottom row: end of presentation ramp. (A) Texture element size expanded twice as quickly as square size. (B) Texture element size and square size both expanded at the same rate. (C) Square size expanded while texture element size remained constant.

The relation between the object's angular subtense $\left(\theta_{t}\right)$ at time $t$ and time $(t)$ is given by the following equation:

$$
\theta_{t} \approx \frac{\theta_{0}}{1-\left(t / T_{\theta}\right)}
$$

(Regan \& Hamstra, 1993), where $\theta_{\mathrm{o}}$ is the object's subtense at time $t=0$ and $T_{\theta}$ is the time to collision at time $t=0$ signaled by the rate of expansion of the object's angular subtense $\theta$.

The separation between the centers of adjacent dots $(x)$ varied according to the following equation:

$$
x_{t}=\frac{x_{0}}{1-\left(t / T_{x}\right)}
$$

where $T_{x}$ was the time to collision signaled by the variation of dot separation.

The angular subtense of the dots $(s)$ varied with time according to the following equation:

$$
s_{t}=\frac{s_{0}}{1-\left(t / T_{s}\right)},
$$

where $T_{s}$ was the time to collision signaled by the variation of dot size.

We report psychophysical data obtained in two conditions. In the first condition, simulation was accurate (i.e., $T_{\theta}=T_{x}=T_{s}$ ). In the second condition, the rate of increase of the object's angular subtense signaled the same time to collision as did the rate of increase of dot separation $\left(T_{\theta}, x\right)$, but $T_{\theta}, x$ was different from the time to collision signaled by the rate of increase of dot subtense $\left(T_{s}\right)$. We define ratio $R$ as follows:

$$
R=\frac{T_{\theta, x}}{T_{s}}=\frac{\theta(\mathrm{d} s / \mathrm{d} t)}{(\mathrm{d} \theta / \mathrm{d} t) s} .
$$

Ratio $R$ could take any value between zero and two. The values of dot size at time $t=0$ (i.e., $s_{0}$ ) and of the separation between adjacent dots at time $t=0$ (i.e., $x_{0}$ ) were chosen so that the dots never overlapped.

\section{Psychophysical Procedure}

Estimates of absolute time to collision were documented by using a tracking procedure developed in previous studies (Gray \& Regan, 1996, 1998). Bearing in mind that repeated exposure to an expanding target produces a temporary decrease in the perceived speed of motion in depth (Regan \& Beverley, 1978a, 1979a), the procedure was designed so that each run contained a number of trials that was sufficiently small that appreciable adaptation was avoided, but not so small that estimates of time to collision were unacceptably variable. Also, the intertrial interval was sufficiently long $(8 \mathrm{sec})$ that adaptation was minimized, yet not so long as to unduly prolong each run. A compromise was reached by trial and error in preliminary experiments. The procedure will first be outlined for the accurate simulation, in which all three types of expansion correspond to the same time to collision (i.e., $R=1.0$ ). In this condition, $\theta, x$, and $s$ all increase identically. Therefore, we need discuss the method only in terms of $\theta$.

Each trial consisted of one presentation of the textured square. At time $t=0$, the square appeared and remained visible for a mean duration of $0.7 \mathrm{sec}$. Presentation duration was varied by $\pm 25 \%$ about the mean on a trial-to-trial basis. At the designated time to collision, some time after the square had been switched off, a brief auditory click was generated. The designated time to collision was set to an accuracy of $0.001 \mathrm{sec}$. The observer was instructed to press one of two buttons, depending on whether the auditory click occurred before or after the simulated approaching textured object would have arrived at the eye.

The time to collision of the simulated approaching object [i.e., $\theta /(\mathrm{d} \theta / \mathrm{d} t)]$ was varied from trial to trial by the computer that controlled the experiment. Before any given trial, the computer set the time to collision of the next presentation on the basis of the observer's previous button presses. This was done according to the staircase method described in detail by Levitt (1971). For clarity, we first describe the procedure for one staircase (though several staircases were interleaved). If the observer indicated that the simulated approaching object would have arrived before the auditory click, the time to collision was made longer for the next presentation in the staircase, and vice versa. Thus, $\theta /(\mathrm{d} \theta / \mathrm{d} t)$ (i.e., the time to collision of the simulated object) might be different on each successive trial. The time to the click (i.e., the designated time to collision) was constant within any given staircase. The initial step size of the staircase (i.e., the amount by which the time to collision $T_{\theta}$ was changed between presentations) was $400 \mathrm{msec}$, and the step size was halved after the first reversal. A reversal occurred when the observer's response changed from before to after, or vice versa. The time to collision on the first presentation in the staircase was chosen randomly before each run. The endpoint of the staircase was based on the final four reversals (the first two or more reversals were ignored). The staircase converged onto a value of $\theta /(\mathrm{d} \theta / \mathrm{d} t)$ that gave a $50 \%$ probability that the observer would judge that the simulated approaching object would arrive before the auditory click. We took the value of $\theta /(\mathrm{d} \theta / \mathrm{d} t)$ at the $50 \%$ convergence point as the observer's estimate of the value of $\theta /(\mathrm{d} \theta / \mathrm{d} t)$ at time $t=0$ that corresponded to the designated time to collision.

Nine staircases were randomly interleaved, and the procedure followed within each staircase was exactly as described above. The reasons for using multiple interleaved staircases were as follows. First, if only one staircase had been used within a given run, the observers could have anticipated the variations in time to collision (e.g., they could have learned that pressing the before button would result in a longer time to collision on the next trial). This is not possible when multiple staircases are randomly interleaved. Second, the use of multiple staircases allowed us to determine which optical variables the observers used to estimate time to collision. Although the observers were instructed to base their responses on the perceived time to collision of the simulated approaching object, in principle they might not have based their responses entirely on the task-relevant variable and might have used the rate of expansion $(\mathrm{d} \theta / \mathrm{d} t)$ and/or the total change in size $(\Delta \theta)$. Indeed, it has been shown that, in some situations (e.g., peripheral vision), time to collision discriminations are not independent of $\mathrm{d} \theta / \mathrm{d} t$ (Regan \& Vincent, 1995). Had we used only one staircase with a constant presentation duration and a constant starting size, the two task-irrelevant variables (namely, $\mathrm{d} \theta / \mathrm{d} t$ and 

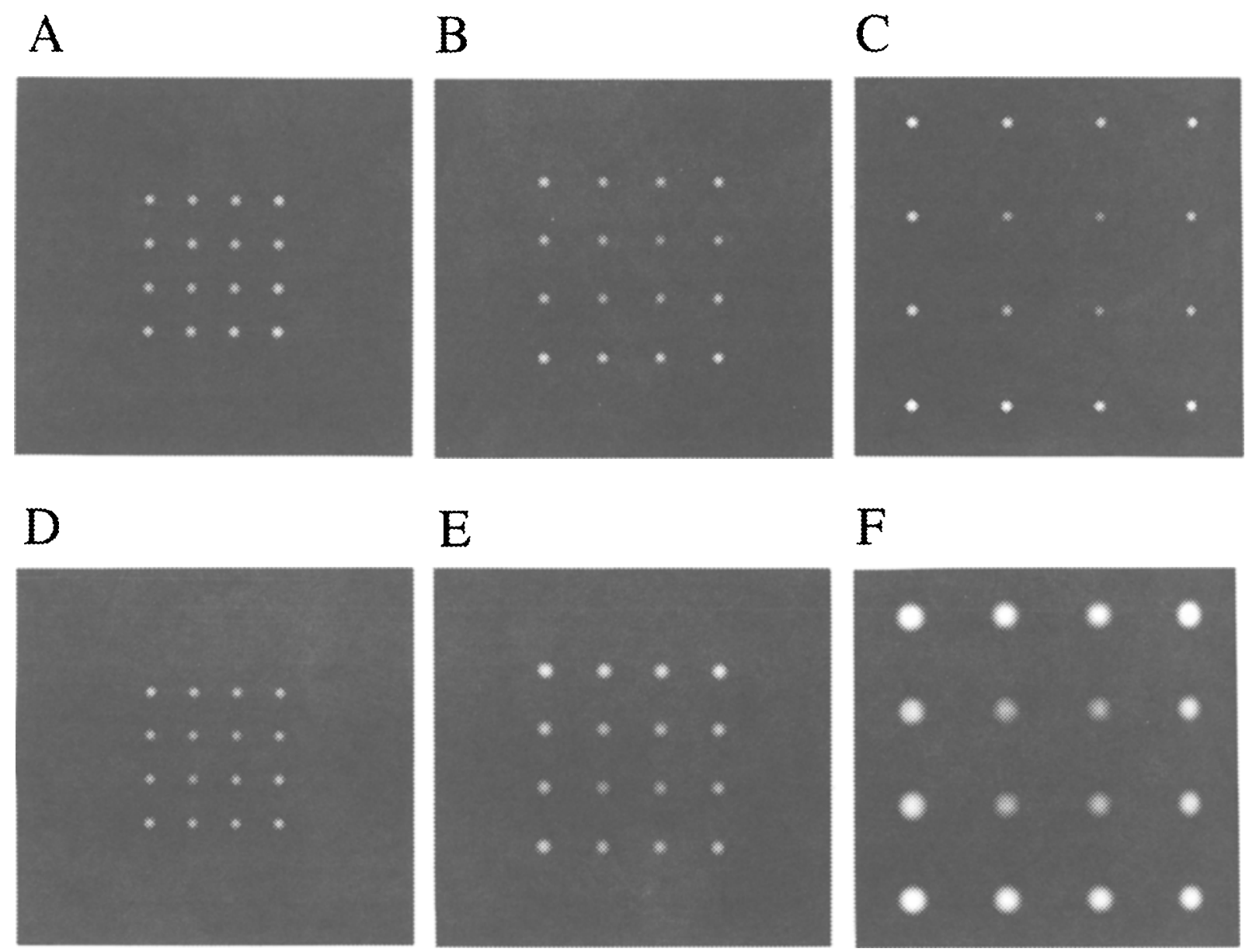

Figure 2. A-C: Photographs of the display for the largest mean dot diameter used (10.5 min of arc at time $t=0)$. A and D: Display at time $t=0$. B and $E$ : Display at time $t=0.35 \mathrm{sec}$. C and F: Display at time $t=0.7 \mathrm{sec}$. A-C: Fractional increase per unit time of target size, dot size, and dot separation were identical. D-F: As for A-C, except that dot size remained constant.

$\Delta \theta$ ) would have been highly correlated with the task-relevant variable $\theta /(\mathrm{d} \theta / \mathrm{d} t)$. We reduced the correlation between the task-relevant variable and these task-irrelevant variables by varying the square's starting size $\left(\theta_{0}\right)$ independently of the designated time to collision. (Recollect that, for the accurately simulated display used in Experiment 1 , the same time to collision is signaled simultaneously by the rates of increase of object size, dot size, and dot separation.) We interleaved three target starting sizes $(22,29$, and $38 \mathrm{~min}$ of arc) and three designated times to collision $(1.8,2.2$, and $2.6 \mathrm{sec})$. The nine staircases correspond to all possible combinations of these two variables. For any given mean dot size, we interleaved three values of dot separation at time $t=0$ and three slightly different values of dot diameter at time $t=0$ in different runs.

\section{Data Analysis}

The accuracy of time-to-collision judgments was quantified by calculating the percentage difference between the designated time to collision and the estimated time to collision for each of the nine staircases in a given run. Each observer completed four runs for each condition, so that the mean percentage error in time-to-collision estimation was based on 36 estimates. ${ }^{1}$

Response data were also subjected to stepwise regression analysis to find the weighting the observers placed on different variables when making time-to-collision judgments. ${ }^{2}$

\section{Observers}

Three observers were used. Observer 1 (author R.G.) was a male 27 years of age. Observers 2 and 3 were females, both 22 years of age. Observers 2 and 3 were naive as to the aims of the experiment and were paid an hourly rate.

\section{EXPERIMENT 1}

\section{Purpose}

The aim of Experiment 1 was to establish a baseline by measuring the absolute accuracy of estimating time to collision with a simulated approaching textured object in the everyday situation in which the fractional increases per unit time of the following three variables were identical: target size $(\theta)$, texture element separation $(x)$, and texture element size $(s)$.

\section{Method}

Procedure. Five mean dot diameters were used $(2.2,4.4,6.0$, 7.6 , and $10.5 \mathrm{~min}$ of arc at time $t=0$ ). Recollect that, for the reasons described in the General Method section, for each mean dot diameter, three slightly different dot diameters were interleaved. Fig- 


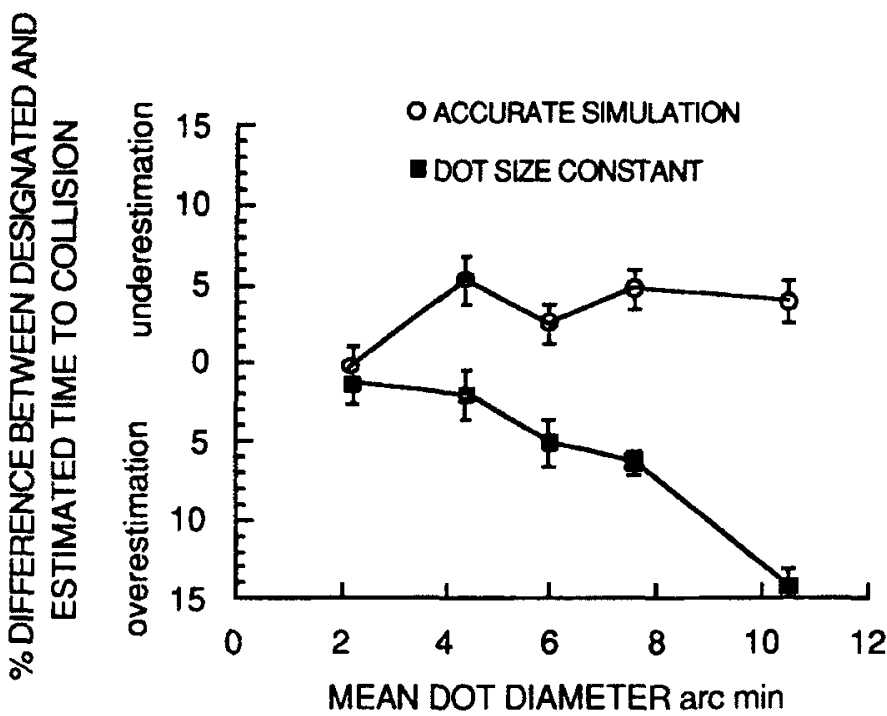

Figure 3. The absolute error in estimating time to collision with a simulated approaching rigid nonrotating textured object was plotted as ordinate versus the initial diameter of the texture dots covering the target. In the first condition, the target accurately simulated an approaching textured object (open symbols). In the second condition, the texture dots covering the target were constant in size (filled symbols). Data points are means for $3 \mathrm{ob}-$ servers.

ures $2 \mathrm{~A}-2 \mathrm{C}$ show the target with the largest dots at times $t=0, t=$ 0.35 , and $t=0.7 \mathrm{sec}$, respectively.

\section{Results}

The open circles in Figure 3 plot the absolute error in estimating time to collision versus the size of the texture dots at time $t=0$. Data points are means for the $3 \mathrm{ob}-$ servers. It is evident that size had no consistent effect on the error and that there was a tendency to underestimate time to collision. The mean error averaged over dot size and observer was $3.2 \%$. Errors ranged from $0.4 \%$ to $10.2 \%$.

Table 1 shows $R^{2}$ values obtained by subjecting the response data to stepwise multiple regression analysis. The dependent variable was always the estimated time to collision. The following independent variables were entered: designated time to collision, $\theta_{0}, x_{0}, s_{0}$, final $\theta$, final $x$, final $s, \Delta \theta, \Delta x, \Delta s, \mathrm{~d} \theta / \mathrm{d} t, \mathrm{~d} x / \mathrm{d} t$, and $\mathrm{d} s / \mathrm{d} t$. For all $3 \mathrm{ob}-$

Table 1

\begin{tabular}{|c|c|c|c|c|c|}
\hline oserver & $\begin{array}{c}\text { Mean } \\
\text { Dot Size }\end{array}$ & $\begin{array}{c}\text { Most } \\
\text { Significant Variable }\end{array}$ & $R^{2}$ & $\begin{array}{c}\text { Next } \\
\text { Significant Variable }\end{array}$ & $R^{2}$ \\
\hline \multirow[t]{5}{*}{ l } & 2.2 & Designated TTC & .95 & NA & $\mathrm{N}$ \\
\hline & 4.4 & Designated TTC & .82 & NA & \\
\hline & 6.0 & Designated TTC & .83 & NA & \\
\hline & 7.6 & Designated TTC & .89 & NA & \\
\hline & 10.5 & Designated TTC & .79 & NA & $\mathrm{N}$ \\
\hline \multirow[t]{5}{*}{2} & 2.2 & Designated TTC & .74 & NA & $\mathrm{N}$ \\
\hline & 4.4 & Designated TTC & .67 & $\Delta \theta$ & .7 \\
\hline & 6.0 & Designated TTC & .69 & NA & $\mathrm{N}$ \\
\hline & 7.6 & Designated TTC & .76 & $\theta_{0}$ & $\mathrm{~N}$ \\
\hline & 10.5 & Designated TTC & .76 & $\theta_{0}$ & .8 \\
\hline \multirow[t]{5}{*}{3} & 2.2 & Designated TTC & .79 & NA & NA \\
\hline & 4.4 & Designated TTC & .72 & $s_{0}$ & 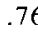 \\
\hline & 6.0 & Designated TTC & .86 & $\theta_{0}$ & .8 \\
\hline & 7.6 & Designated TTC & .89 & NA & $\mathrm{N}$ \\
\hline & 10.5 & Designated TTC & .91 & NA & N \\
\hline
\end{tabular}

Note--TTC, time to collision; NA, not applicable; $\Delta \theta$, change of target's angular size during presentation; $\theta_{0}$, angular size of target at time $t=0 ; s_{0}$, dot diameter at time $t=0$. 


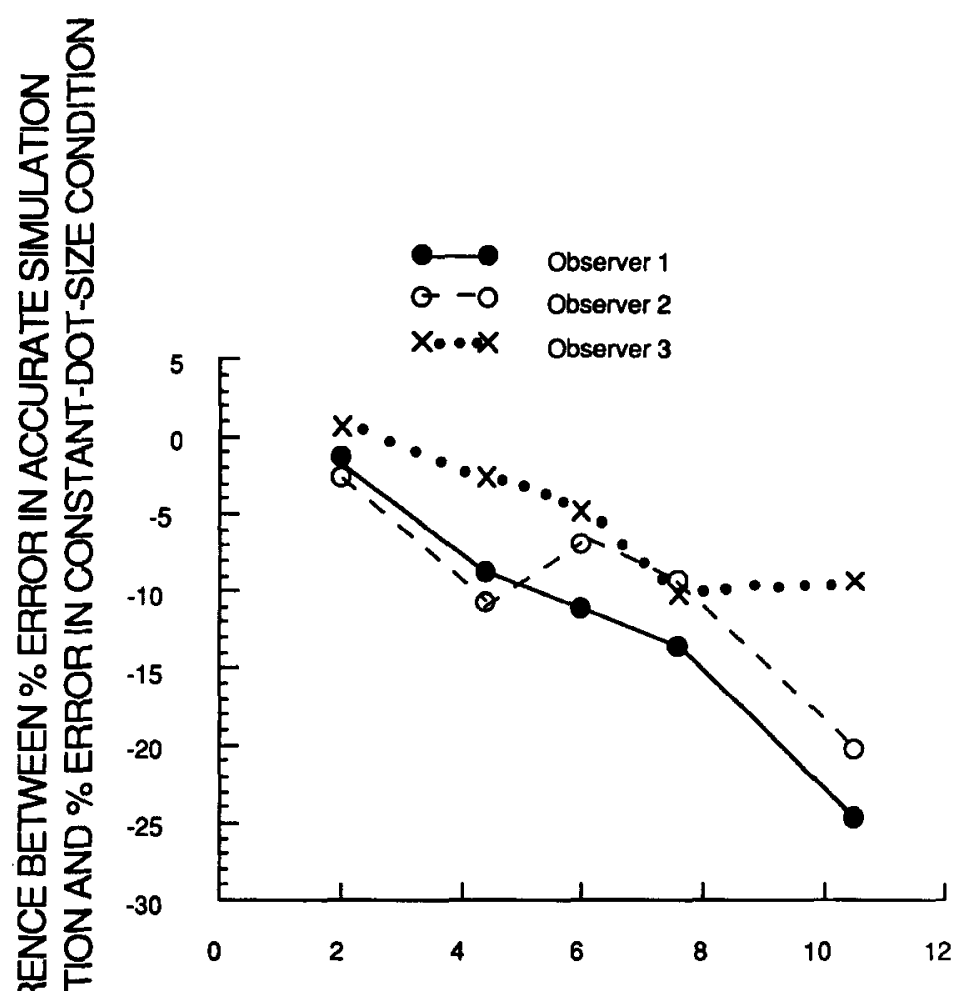

\section{MEAN DOT DIAMETER arc min}

Figure 4. The difference in the absolute error in estimating time to collision between the condition in which simulation was accurate and the condition in which dot size was constant was plotted as ordinate versus dot diameter. This figure brings out interobserver variability.

servers, the task-relevant variable (designated time to collision) accounted for a high proportion of the total variance ( $R^{2}$ ranged from .67 to .91$)$. Although $\theta_{0}, \Delta \theta$, and $s_{0}$ accounted for a significant additional amount of variance in some cases, the amount added was small, ranging from .03 to .12 .

\section{EXPERIMENT 2}

\section{Purpose}

The aim of Experiment 2 was to measure the absolute accuracy of estimating time to collision with a simulated textured object in the situation in which the fractional increases per unit time in target size $(\theta)$ and texture element separation $(x)$ were identical but texture element size $(s)$ remained constant.

\section{Method}

Procedure and Data Analysis. The procedure was the same as that for Experiment 1, except that dot size was constant. Data analysis was the same.

Observers' task. The observers' task was to match the time to collision $\left(T_{\theta}, x\right)$, signaled by both object size and dot separation
(Equations 2 and 3), to the time to the click. The time to collision signaled by dot size was infinite (Equation 4 ).

\section{Results}

The filled symbols in Figure 3 show that, for the largest dot size used, the small underestimation of time to collision observed in Experiment 1 became a considerable overestimation $(7.5 \%-21 \%)$ when dot size was held constant. This effect of holding dot size constant was significant at the $p<.001$ level for all 3 subjects [Observer 1 , $t(70)=10.2$; Observer $2, t(70)=5.3$; Observer $3: t(70)=$ $10.1]$.

Figure 4 brings out the difference between the results of Experiments 1 and 2 on a subject-by-subject basis. For the smallest dot size used, the difference between the results of the two experiments was insignificant [Observer $1, t(70)=0.61, p>.5$; Observer $2, t(70)=1.29, p>$ .2]. For Observer 3 , the difference was insignificant for the two smallest dot sizes [ $2.2 \mathrm{~min}$ of arc dots, $t(70)=$ $0.44, p>.5 ; 4.4 \mathrm{~min}$ arc dots, $t(70)=1.27, p>.2]$.

Table 2 shows $R^{2}$ values obtained by subjecting the data to stepwise regression analysis. Because dot size was 
Table 2

$R^{2}$ Values From Stepwise Multiple Regression Analysis

of Observers' Estimates of Time to Collision When Dot Size Remained Constant

\begin{tabular}{cccccc}
\hline & Mean & Most & \multicolumn{2}{c}{ Next } \\
Observer & Dot Size & Significant Variable & $R^{2}$ & Significant Variable & $R^{2}$ \\
\hline 1 & 2.2 & Designated TTC & .93 & $x_{0}$ & .94 \\
& 4.4 & Designated TTC & .70 & NA & NA \\
& 6.0 & Designated TTC & .81 & NA & NA \\
& 7.6 & Designated TTC & .96 & NA & NA \\
2 & 10.5 & Designated TTC & .72 & NA & NA \\
& 2.2 & Designated TTC & .67 & $\theta_{0}$ & .72 \\
& 4.4 & Designated TTC & .65 & $\Delta \theta$ & .71 \\
& 6.0 & Designated TTC & .71 & Final $\theta$ & .76 \\
& 7.6 & Designated TTC & .68 & d $x / \mathrm{d} t$ & .70 \\
3 & 10.5 & Designated TTC & .73 & NA & NA \\
& 2.2 & Designated TTC & .90 & NA & NA \\
& 4.4 & Designated TTC & .80 & $x_{0}$ & .92 \\
& 6.0 & Designated TTC & .82 & NA & .85 \\
& 7.6 & Designated TTC & .83 & NA & NA \\
& 1.5 & Designated TTC & .79 & NA & NA \\
\hline
\end{tabular}

Note-TTC, time to collision; $x_{0}$, dot separation at time $t=0$; NA, not applicable; $\theta_{0}$, target size at time $t=0 ; \mathrm{d} x / \mathrm{d} t$, rate of change of dot separation at time $t=0 ; s_{0}$

held constant, it was not necessary to enter the final dot size, the change in dot size, or the rate of change of dot size. Otherwise, the same variables were entered as those in Experiment 1. The designated time to collision accounted for a high proportion of the total variance $\left(R^{2}\right.$ ranged from .65 to .96$)$. The amount of additional variance explained by the other variables was small.

A repeated measures analysis of variance (ANOVA) showed that dots expanding versus dot size constant was significant $[F(1,2)=21.3, p<.05]$, as was the interaction between dots expanding versus dot size constant and dot size $[F(4,8)=11.6, p<.01]$.

\section{GENERAL DISCUSSION}

As was reported previously, when simulation is accurate, observers usually underestimate the time to collision (Cavallo \& Laurent, 1988; Gray \& Regan, 1998; Schiff $\&$ Detwiler, 1979). The only exception in 15 cases was Observer 2 for the smallest starting size (2.2 $\mathrm{min}$ of arc diameter dots).

Our new data lead to the conclusion that the error in estimating the absolute time to collision with an approaching simulated textured object can be considerably increased when texture element size is held constant, rather than expanding in accord with the expansion of target size. The effect of holding dot size constant is in the direction of overestimation of time to collision (a sufficiently large overestimation would mean that measures taken to avoid collision would be too late). This effect decreases as dot size is decreased and becomes insignificant at sufficiently small dot sizes. The absence of an effect at small dot sizes presumably occurs because, as quantified previously (Regan \& Beverley, 1979a; Gray \& Regan, 1998), for any given time to collision, a dot's rate of change of size in deg/sec grows progressively less (and, therefore, stimulation becomes progressively weaker) as dot size is reduced.

In a series of papers, we have developed a psychophysical model of the visual processing of retinal image expansion and the consequent generation of motion-indepth perception (Beverley \& Regan, 1979a, 1979b, 1980, 1983; Gray \& Regan, 1998; Regan \& Beverley, 1978a, 1978b, 1979a, 1979b, 1980, 1981; Regan \& Hamstra, 1993; Regan \& Vincent, 1995). Figure 5 depicts a recent version. In brief, the dashed line indicated the boundaries of an expanding untextured retinal image. The instantaneous speed and direction of motion of each edge is encoded by filters sensitive to local motion (LM). These we assume to be elaborated Reichardt detectors (Santen \& Sperling, 1985). Relative motion (RM) filters encode the difference in the velocities of opposite edges (Regan \& Beverley, 1978b, 1980). The motion-in-depth filter (MID) outputs a motion-in-depth signal after testing for isotropic expansion. In Figure 5, the test for isotropic expansion is that

$$
\frac{\theta_{\mathrm{H}}}{\left(\mathrm{d} \theta_{\mathrm{H}} / \mathrm{d} t\right)}=\frac{\theta_{\mathrm{V}}}{\left(\mathrm{d} \theta_{\mathrm{V}} / \mathrm{d} t\right)}
$$

that is, that the increases of the vertical $\left(\theta_{\mathrm{v}}\right)$ and horizontal $\left(\theta_{\mathrm{H}}\right)$ dimensions of the retinal image signal the same time to collision. [Note that the ratios $\theta_{\mathrm{H}} /\left(\mathrm{d} \theta_{\mathrm{H}} / \mathrm{d} t\right)$ and $\theta_{\mathrm{v}} /\left(\mathrm{d} \theta_{\mathrm{v}} / \mathrm{d} t\right)$ are, respectively, the times to collision signaled by the rate of expansion of the object's horizontal and vertical diameters.] Thus, time to collision is computed before the motion-in-depth signal is generated. We have reported psychophysical evidence for this processing stage (Beverley \& Regan, 1979a, 1980). We modeled sensitivity to this two-dimensional relationship by assuming that the value of $k$, for any given RM filter, is inversely pro- 


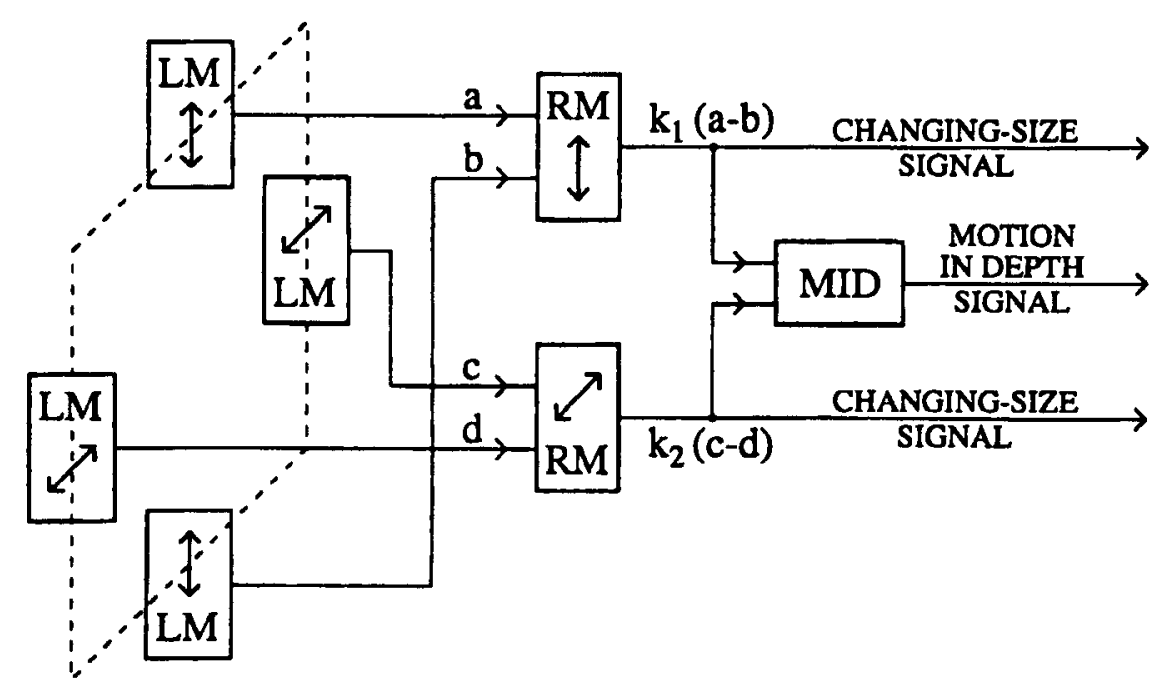

Figure 5. Model of the processing of changing size and of the encoding of time to collision for an untextured object. The retinal image boundary is shown dotted. $L M$ represents filters sensitive to local motion along the directions arrowed. Their outputs $(a, b, c$, and $d)$ assume a magnitude that encodes local speed and a sign that represents the direction of motion. $R M$ represents one-dimensional relative motion filters whose outputs signal the speed and sign (expansion vs. contraction) of relative motion along some given retinal meridian. $M I D$ represents a twodimensional relative motion filter for which the most efficient stimulus is isotropic expansion of the retinal image - that is, the situation that $k_{1}(a-b)=k_{2}(c-d)$. We assume here that the amplitude of the output of the MID filter is equal to that of any one of its inputs from the RM filters. If so, this output is inversely proportional to time to collision. From "Dissociation of Discrimination Thresholds for Time to Collision and for Rate of Angular Expansion," by D. Regan and S. J. Hamstra, 1993, Vision Research, 33, p. 457. Copyright 1993 by Elsevier Science. Reprinted with permission.

portional to the angular separation of the two LM filters that feed it-that is, $k_{1}=K / \theta_{\mathrm{v}}$ and $k_{2}=K / \theta_{\mathrm{H}}$, where $K$ is a constant. In this case, Equation 6 is satisfied for an isotropically expanding retinal image, and, furthermore, the output of each RM filter is inversely proportional to time to collision.

We have reported evidence that, for retinal image locations within a few degrees of the fovea, the human visual system contains a mechanism that is sensitive to trial-totrial variations in the ratio $\theta /(\mathrm{d} \theta / \mathrm{d} t)$ and is, therefore, sensitive to trial-to-trial variations in time to collision (Equation 1) independently of trial-to-trial variations in image size $(\theta)$ and rate of expansion $(\mathrm{d} \theta / \mathrm{d} t)$ (Regan \& Hamstra, 1993; Regan \& Vincent, 1995). We have further proposed that the perceived speed of an approaching object's motion in depth is determined, not by the object's actual speed, but rather by the reciprocal of the time to collision (Regan \& Hamstra, 1993). This proposal is consistent with the findings that adapting to a rate of expansion both reduces the perceived speed of motion in depth and converts small underestimations to substantial overestimations of time to collision (Gray \& Regan, in press).

Our present finding can be explained by extending our previous model as indicated in Figure 6 . The circle at the upper left in Figure 6 indicates the boundary of a single texture element. Two pairs of LM filters that are tuned to horizontal and vertical motion, respectively, encode the velocities of the edges perpendicular to the texture element's horizontal $\left(s_{\mathrm{H}}\right)$ and vertical $\left(s_{\mathrm{V}}\right)$ angular diameters, and these LM filters feed RM filters that encode the rates of expansion of the texture element's horizontal and vertical diameters. (In our present case, the texture elements are circular dots, so that $s_{\mathrm{H}}=s_{\mathrm{v}}=s$, and the horizontal and vertical diameters expand identically.) Following Figure 5, we assume in Figure 6 that $k_{1}=k_{2}=K / \mathrm{s}$, so that the outputs of the two top RM filters are each inversely proportional to the time to collision signaled by the rate of dot expansion. We assume that the expansion of every texture dot in the display is processed similarly. We also assume that the outputs of all the vertical RM filters are averaged, as are the outputs of all the horizontal RM filters. To avoid unnecessary clutter in Figure 6, the processing of the expansion of an individual dot is shown only for the uppermost dot.

As was just mentioned, the uppermost two RM filters shown in Figure 6 and all the other RM filters driven by the expansion of individual dots receive inputs from pairs of LM filters that are separated by a distance that is approximately equal to the diameter of one texture dot $^{3} \mathrm{We}$ now propose that other RM filters receive inputs from $\mathrm{LM}$ 


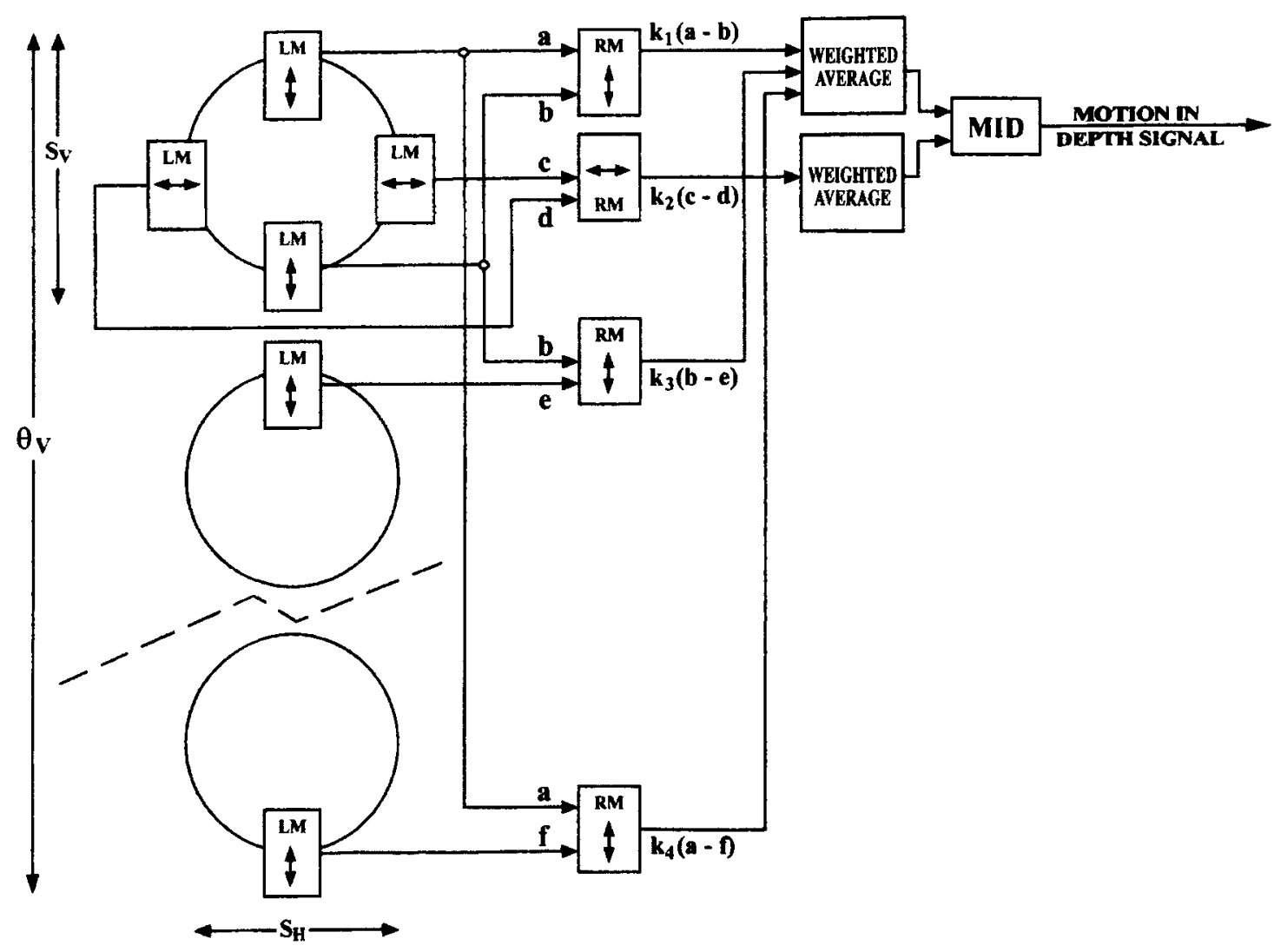

Figure 6. Model of the processing of changing size and the encoding of time to collision for a textured object. The object's textured retinal image subtends $\theta_{\mathrm{v}}$ vertically and $\theta_{\mathrm{H}}$ horizontally. For clarity, only the horizontal dimension is shown. The bright texture elements (circles at the left of the figure) subtend $s_{\mathrm{v}}$ vertically and $s_{\mathrm{H}}$ horizontally. Although bright texture elements are shown as being circular and all of the same size, neither of these conditions is necessary. The topmost two dots are adjacent. The top and bottom dots demarcate the upper and lower boundaries of the object's retinal image. The jagged dashed line indicates that several dots may lie between the bottom and the middle dots. LM, RM, and MID filters are the same as those in Figure 5. See text for details.

filters that are separated by distances up to and including the diameter of the retinal image of the entire simulated object.

The processing of changes in the angular size of the entire object is illustrated in Figure 6, where the upper circle represents a dot on the upper edge of the object's retinal image and the lower circle represents a dot on the lower edge of the object's retinal image. (The jagged dashed line indicates that there may be several dots between the lowest dot and the middle dot.) The rate of expansion of the vertical diameter of the object's retinal image (i.e., $\mathrm{d} \theta_{\mathrm{v}} / \mathrm{d} t$ ) is signaled by the lowermost RM filter in Figure 6 . If we assume that $k_{4}=K / \theta_{\mathrm{v}}$, the output of the lowermost RM filter will be inversely proportional to the time to collision signaled by the rate of expansion of the vertical diameter of the object's retinal image [i.e., inversely proportional to $\left.\theta_{\mathrm{v}} /\left(\mathrm{d} \theta_{\mathrm{v}} / \mathrm{d} t\right)\right]$. We assume that the rate of expansion of the horizontal diameter of the object's retinal image is processed similarly, but to avoid undue clutter, this connectivity is not shown in Figure 6.

The two uppermost dots in Figure 6 are adjacent. The third RM filter from the top signals the rate of expansion of the black region between the two dots. If we assume that $k_{3}=K / \alpha$, the output of this filter is inversely proportional to the time to collision signaled by the rate of expansion of this black region (where $\alpha$ is the vertical angular subtense of the black region). ${ }^{4}$ As is shown in Figure 6 , this signal is averaged with all the other signals from vertical RM filters. We assume that the rate of change of the horizontal dimensions of black regions between dots is signaled similarly, but to avoid undue clutter, this connectivity is not shown in Figure 6.

Next, we compare the predictions of this model with our previous experimental findings. In the accurate-simulation condition used in previous studies (Figure 1B), the outputs of all the RM filters signaled the same time to collision, and we found that the motion-in-depth signal was strong (Beverley \& Regan, 1983). We also found that errors in estimating the time to collision were small (Vincent \& Regan, 1997). When object size expanded while textureelement size remained constant, the motion-in-depth signal was reduced by $36 \%$ and $42 \%$, respectively, for $2 \mathrm{ob}-$ servers (Beverley \& Regan, 1983), and the time to collision signaled by the expansion of object size was overesti- 
mated by $22 \%$ and $36 \%$, respectively, for 2 observers. ${ }^{5}$ This suggests that the outputs of the RM filters were averaged in a way that gave greater weight to the expansion of object size than to the (lack of) expansion of texture element size. When texture element size expanded at twice the rate of that for object size (Figure $1 \mathrm{~A}$ ), the motionin-depth signal was only $42 \%$ stronger than in the case of accurate simulation (Beverley \& Regan, 1983), and the time to collision signaled by the expansion of object size was estimated to be only $13 \%$ and $14 \%$ ( 2 subjects) less than that in the case of accurate simulation (Vincent \& Regan, 1997). Again, this suggests that averaging gave greater weight to object expansion than to the expansion of texture elements.

Next, we compare the predictions of our model with our present experimental findings. In the condition of accurate simulation (Experiment 1, Figures $2 \mathrm{~A}-\mathrm{C}$ ), our present stimulus is comparable with the stimulus used previously (Figure 1B) in that identical times to collision were signaled by the expansion of the object, by the expansion of the bright texture elements, and by the expansion of the dark regions between bright texture elements; the model shown in Figure 6 predicts our present findings along the lines just discussed.

The two stimuli are not, however, comparable in the condition in which object size expands while the sizes of the bright texture elements remains constant. For the stimulus shown in Figure 1B, both the bright texture elements and the dark regions between texture elements are of constant size, so that the classes of RM filters exemplified by the top three in Figure 6 all signal an infinite time to collision. For our present stimulus, however, the black regions between dots expand at the same rate as the object (Figures 2D-2F), so that the class of RM filters exemplified by the third from the top in Figure 6 will signal the same time to collision as the lowermost RM filter. Our model predicts that the perceived speed of motion in depth would be weaker than it would in the case of accurate simulation and that the estimate of time to collision would be longer than it would in the case of accurate simulation. ${ }^{6}$ These predictions accord with our findings.

For completeness, we should state the prediction for the condition in which object size increases while both dot size and dot separation remain constant. This stimulus is comparable with the stimulus used previously (Figure 1C), in that all the RM filters of the classes exemplified by the topmost three in Figure 6 will signal an infinite time to collision, and only the class exemplified by the lowest RM filter in Figure 6 will signal a finite time to collision. In accord with this prediction, our observers reported that the sensation of motion in depth produced by this stimulus was either very weak or totally absent; consequently, no observer was able to carry out the experimental procedure to estimate time to collision.

Finally, as mentioned earlier, many authors have used patterns of constant-sized dots to collect data on the perception of motion in depth, on the perception of self- motion, and on judgements of heading. Our findings suggest that the relevance of such data to everyday visual perception might be questionable if dot size exceeds 2.2-4.4 min of arc, depending on the particular observer.

\section{REFERENCES}

BeverLey, K. I., \& Regan, D. (1979a). Separable aftereffects of changing-size and motion-in-depth: Different neural mechanisms? Vision Research, 19, 727-732.

BEVERLEY, K. I., \& REGAN, D. (1979b). Visual perception of changingsize: The effect of object size. Vision Research, 19, 1093-1104.

BEVERLEY, K. I., \& REGAN, D. (1980). Visual sensitivity to the shape and size of a moving object: Implications for models of object perception. Perception, 9, 151-160.

BeverLey, K. I., \& Regan, D. (1983). Texture changes versus size changes as stimuli for motion in depth. Vision Research, 23, 13871400 .

CaVAllo, V., \& Laurent, M. (1988). Visual information and skill level in time-to-collision estimation. Perception, 17, 623-632.

GRAY, R., \& REGAN, D. (1996). Estimates of time to collision based on binocular and monocular visual information. Investigative Ophthalmology \& Visual Science, 37, S652.

Gray, R., \& REGAN, D. (1998). Accuracy of estimating time to collision using binocular and monocular information. Vision Research, 38, 499-512.

GRAY, R., \& REGAN, D. (in press). Adapting to expansion increases perceived time to collision. Vision Research.

HoYLE, F. (1957). The black cloud. London: Penguin.

LEE, D. N. (1976). A theory of visual control of braking based on information about time-to-collision. Perception, 5, 437-459.

LEVITT, H. (1971). Transformed up-down methods in psychoacoustics. Journal of the Acoustical Society of America, 49, 65-69.

REGAN, D., \& BEVERLEY, K. I. (1978a). Illusory motion in depth: Aftereffect to adaptation to changing size. Vision Research, 18, 209-212.

Regan, D., \& BeVerLey, K. I. (1978b). Looming detectors in the human visual pathway. Vision Research, 18, 415-421.

REGAN, D., \& BEVERLEY, K. I. (1979a). Binocular and monocular stimuli for motion in depth: Changing-disparity and changing-size feed the same motion-in-depth stage. Vision Research, 19, 1331-1342.

Regan, D., \& BeverLey, K. I. (1979b). Visually guided locomotion: Psychophysical evidence for a neural mechanism sensitive to flow patterns. Science, 205, 311-313.

REGAN, D., \& BeverLeY, K. I. (1980). Visual responses to changing size and to sideways motion for different directions of motion in depth: Linearization of visual responses. Journal of the Optical Society of America, 70, 1289-1296.

REGAN, D., \& BeVERLEY, K. I. (1981). Motion sensitivity measured by a psychophysical linearizing technique. Journal of the Optical Society of America, 71, 958-965.

Regan, D., \& Hamstra, S. J. (1993). Dissociation of discrimination thresholds for time to contact and rate of angular expansion. Vision Research, 33, 447-462.

REGAN, D., \& VinCENT A. (1995). Visual processing of looming and time to contact throughout the visual field. Vision Research, 35, 1845-1857.

SANTEN, J. P. H., \& Sperling, G. (1985). Elaborated Reichardt detectors. Journal of the Optical Society of America A, 2, 300-321.

SCHIFF, W., \& DeTWILER, M. L. (1979). Information judged in impending collision. Perception, 8, 647-658

VINCENT, A., \& REGAN, D. (1997). Judging the time to collision with a simulated textured object: Effect of mismatching rate of expansion of object size and of texture element size. Perception \& Psychophysics, 59, 32-36.

\section{NOTES}

1. This is the mean of the percentage errors signed for underestimation and overestimation. For brevity, we will follow a reviewer's suggestion and call it the constant error. 
2. The following is from the handbook provided with the Statview II (Abacus Concepts Inc., Berkeley, CA) package that we used.

Statview computes a multiple linear regression using the forward stepwise regression with elimination of unnecessary variables. The forward selection procedure selects as the next variable for the regression model that independent variable with the highest partial correlation with the dependent variable.

Essentially, the partial F-ratio associated with each remaining variable is computed based upon the inclusion of a remaining variable into the existing equation. Of those variables not included in the regression equation, that variable with the largest partial F-ratio is selected for inclusion and then new partial F-ratios are computed.

3. Evidence that any given RM detector is fed by a pair of LM filters that have some unique separation has been reported previously (Beverley \& Regan, 1979b, 1980).

4. We have previously shown that the illusory perception of motion in depth created by inspecting a bright-on-dark target whose size is changing transfers to a test target of reversed contrast- - that is, darkon-bright (Regan \& Beverley, 1978a).

5 . These were not the same 2 observers who participated in the 1983 study.

6. Our model also predicts that the increase in estimated time to collision would be smaller in the present case than the increase reported by Vincent and Regan (1997). Although this seems to be the case ( $7 \%-21 \%$, depending on dot size, mean of 3 observers, in the present case, as compared with $22 \%$ and $36 \%$ for 2 observers in the 1997 paper). comparison is difficult because different observers were used in the two studies.

(Manuscript received September 29, 1997; revision accepted for publication February 13, 1998.) 